\title{
Estimating Dynamic Gait Stability Using Data from Non-aligned Inertial Sensors
}

\author{
Sjoerd M. Bruijn, ${ }^{1}$ Warner R. Th. Ten Kate, ${ }^{2}$ Gert S. Faber, ${ }^{1}$ Onno G. Meijer, ${ }^{1,3}$ Peter J. Beek, ${ }^{1}$ \\ and JAAP H. van DieËN ${ }^{1}$ \\ ${ }^{1}$ Research Institute MOVE, Faculty of Human Movement Sciences, VU University Amsterdam, Van der Boechorststraat 9, \\ NL-1081 BT Amsterdam, The Netherlands; ${ }^{2}$ Philips Research, Eindhoven, The Netherlands; and ${ }^{3}$ Second Affiliated Hospital of \\ Fujian Medical University, Quanzhou, Fujian, P.R. China
}

(Received 22 September 2009; accepted 17 March 2010; published online 31 March 2010)

Associate Editor Sean S. Kohles oversaw the review of this article.

\begin{abstract}
Recently, two methods for quantifying the stability of a dynamical system have been applied to human locomotion: local stability (quantified by finite time maximum Lyapunov exponents, $\lambda_{\mathrm{s}}$ and $\lambda_{\mathrm{L}}$ ) and orbital stability (quantified by maximum Floquet multipliers, MaxFm). In most studies published to date, data from optoelectronic measurement systems were used to calculate these measures. However, using wireless inertial sensors may be more practical as they are easier to use, also in ambulatory applications. While inertial sensors have been employed in some studies, it is unknown whether they lead to similar stability estimates as obtained with optoelectronic measurement systems. In the present study, we compared stability measures of human walking estimated from an optoelectronic measurement system with those calculated from an inertial sensor measurement system. Subjects walked on a treadmill at three different speeds while kinematics were recorded using both measurement systems. From the angular velocities and linear accelerations, $\lambda_{\mathrm{s}}, \lambda_{\mathrm{L}}$, and MaxFm were calculated. Both measurement systems showed the same effects of walking speed for all variables. Estimates from both measurement systems correlated high for $\lambda_{\mathrm{s}}$ and $\lambda_{\mathrm{L}}$, $(R>0.85)$ but less strongly for MaxFm $(R=0.66)$. These results indicate that inertial sensors constitute a valid alternative for an optoelectronic measurement system when assessing dynamic stability in human locomotion, and may thus be used instead, which paves the way to studying gait stability during natural, everyday walking.
\end{abstract}

Keywords-Gait stability, Treadmill walking, Lyapunov exponents, Floquet multipliers, Inertial sensors.

Address correspondence to Jaap H. van Dieën, Research Institute MOVE, Faculty of Human Movement Sciences, VU University Amsterdam, Van der Boechorststraat 9, NL-1081 BT Amsterdam, The Netherlands. Electronic mail: j.vandieen@fbw.vu.nl

\section{INTRODUCTION}

With their high incidence and associated costs, falls form a formidable problem in modern society. ${ }^{26}$ Consequently, there is a rapidly growing body of research focusing on the (in) stability of posture and gait in the elderly and various patient groups.

Recently, two "dynamical systems" methods for quantifying stability have been applied in the study of human locomotion: local dynamic stability ${ }^{1,2,4,8,17}$ and orbital stability. ${ }^{1,14,15}$ Local dynamic stability is estimated by means of the maximal finite time Lyapunov exponent (also called divergence coefficient), which quantifies how the system responds continuously to very small perturbations. ${ }^{25}$ Orbital stability assumes strict periodicity and is estimated by means of maximum Floquet multipliers, which quantify how the system responds to very small perturbations in a discrete, stroboscopic manner, i.e. from one cycle to the next. ${ }^{14}$ Note that a non-linear system may be locally unstable, in that neighboring trajectories tend to diverge, while being orbitally stable, in that all trajectories return to a limit cycle, thus preserving the overall periodicity of the gait pattern. Both measures have a sound mathematical basis ${ }^{14,25}$ and have frequently been used to quantify dynamic gait stability. ${ }^{1,2,13,16,20,22}$ Still, their relationship to more real-life notions of stability is not yet clear. ${ }^{11,28}$ To further study this relationship, large studies in which these measures are correlated to real-life notions of stability such as the number of falls will be required.

In most studies published to date, data from optoelectronic measurement systems were used to calculate local dynamic and orbital stability. However, when aiming at the measurement of large populations, it may 
be more practical to use wireless inertial sensors instead of an optoelectronic measurement system, as has been done in some studies (e.g. Refs. 6, 30). There are, however, two essential differences between data obtained from an optoelectronic system and data obtained from an inertial sensing system. The first is that the former measures position, whereas the latter measures linear acceleration and/or angular velocity. The second difference is that the data are expressed in different coordinate systems: a global and a local coordinate system which are not related through an affine transformation (the global system is an inertial system, whereas the local system is not).

Theoretically, using these different signals should lead to the same results when estimating stability measures since the recorded signals represent observations from the same underlying dynamic process. Indeed, when derived from a state space description with enough dimensions, the stability measures in question have been demonstrated to be invariant for the state space description chosen. ${ }^{12,21}$ Thus, first expressing the sensor data in a global coordinate system seems unnecessary, which, given the complexity ${ }^{24}$ and the problems associated with this operation, ${ }^{3}$ would give inertial sensors a distinct advantage over optoelectronic sensors in studying gait stability in both clinic and field.

Nevertheless, a direct comparison between estimates of Lyapunov exponents and Floquet multipliers obtained from optoelectronic data and from nonaligned inertial sensor data has, to our knowledge, not been performed to date. Given the essential differences between the two measurement systems mentioned above, it is not certain that they will yield similar or even comparable results in practice. The shape of the "attractor" (i.e., the sub-space in which signal trajectories reside) will be very different for both methods due to projections of gravitational accelerations, as well as projections of rotations and accelerations onto axes of different coordinate systems, which are related in a time-varying manner to the motion itself (see Fig. 1).

In the present study, we therefore examined the effects of using different state space descriptions (i.e. accelerations and rotational velocities, in global and local coordinate systems) obtained from signals recorded by means of both optoelectronic and inertial sensor systems.

\section{METHODS}

Nine healthy male volunteers participated in the study. A neoprene band with 3 infrared LEDs, used for movement registration with an optoelectronic system (Optotrak, NDI, Canada), was attached to the thorax, over the spine at the level of Th8. Underneath this band, a wireless inertial sensor node (PI-node, Philips, The Netherlands, see Fig. 2) was placed, ${ }^{29}$ consisting of 3D gyroscopes, magnetometers, and accelerometers (in the current study only the accelerometer and gyroscope were used). These sensors are small $(36 \times 56 \times 19 \mathrm{~mm})$ and light weight $(38 \mathrm{~g})$ and use the ZigBee transmission protocol $(2.4 \mathrm{GHz})$ to connect to a PC. The accuracy of these sensors has been tested before and was found to be good. ${ }^{29}$

Subjects walked on a treadmill at 3 different speeds $(0.56,1.12$, and $1.68 \mathrm{~m} / \mathrm{s})$, applied in random order, for a period of $5 \mathrm{~min}$ at each speed. Sample rate was 50 samples per second. The measurement systems were

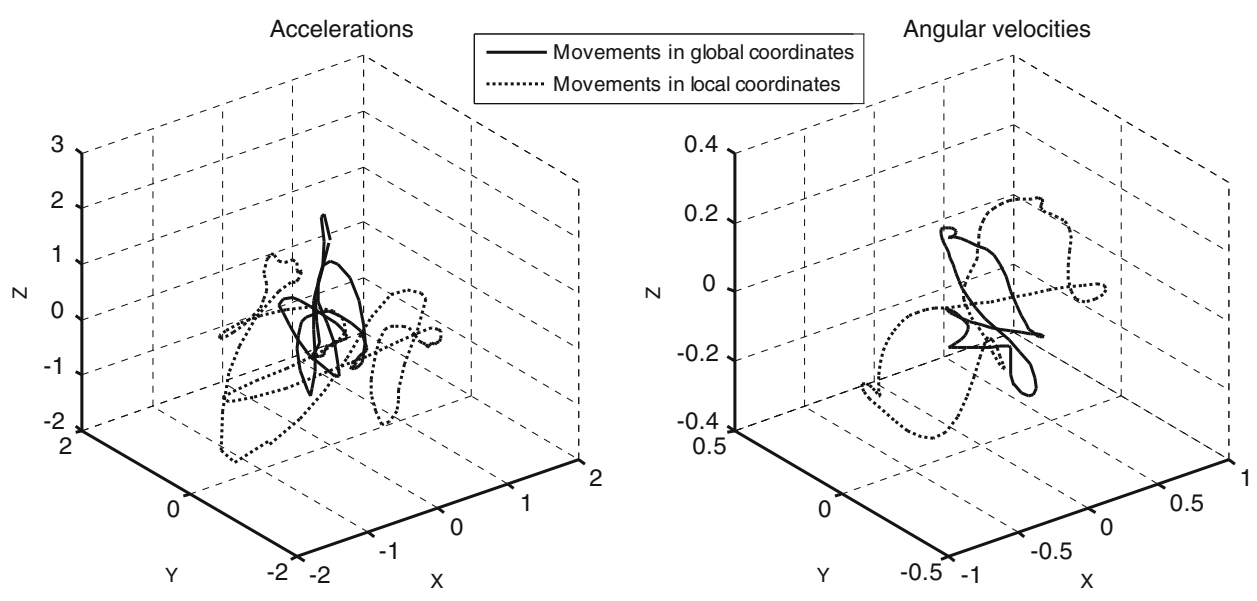

FIGURE 1. Attractors in different reference frames. Attractors constructed from 3D acceleration patterns (left) and rotational velocity (right) during walking, in global (solid trajectories, optoelectronic measurement system) and local (dashed trajectories, inertial sensor measurement) coordinate systems. Note that due to the inclusion of the gravitational acceleration in the inertial sensor acceleration signals, the corresponding attractor is much larger than that of the optoelectronic accelerations. 


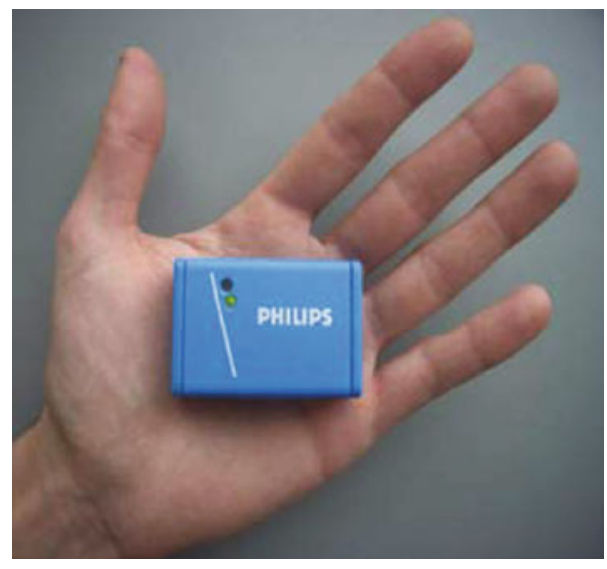

FIGURE 2. Inertial sensor unit. The wireless inertial sensor, which contains accelerometers, magnetometers, and gyroscopes. Note that for the current study only the data from the accelerometers and gyroscopes were used.

not synchronized, but data collection was started at approximately the same time. However, post-hoc, data were synchronized using cross-correlations on the norm of the 3D acceleration signals.

Given the unwanted effects of filtering nonlinear signals, ${ }^{21,23}$ data were analyzed without filtering. Acceleration data were obtained from the optoelectronic position data by double differentiation of the average position of the three markers. Rotational velocities of the optoelectronic data were obtained by a standard procedure (Ref. 31, p. 183, equation 3.46). For both measurement systems, stride cycles were defined as the time between each second peak in the norm of the 3D acceleration signals, which were detected automatically and checked visually. For all time series, the first 150 strides were analyzed and time was normalized so that each time series consisted of 15,000 data points and each stride of approximately 100 samples. ${ }^{1,10}$

Subsequently, 12D state spaces ${ }^{8,17-19}$ were reconstructed from the time-normalized 3D acceleration and $3 \mathrm{D}$ rotational velocities time series, each with their 25 samples delayed copies. Note that the choice of embedding dimension and delay are in principle arbitrary as long as the delay is "reasonable" and the embedding dimension is "sufficiently large" (Ref. 8, p. 1725).

From the state spaces, maximum Lyapunov exponents, which express the sensitivity to small perturbations in real time, were estimated as the slope of the average logarithmic divergence of two initially nearest neighbors ${ }^{25}$ :

$$
y(i)=\frac{1}{\Delta t}\left\langle\ln \left(d_{j}(i)\right)\right\rangle
$$

where $d_{j}(i)$ is the Euclidean distance between the $j$ th pair of nearest neighbors after $i$ discrete time steps $\Delta t$ and $\langle\ldots\rangle$ denotes the average over all values of $j$. This slope was estimated at $0-0.5$ strides $\left(\lambda_{\mathrm{s}}\right)$ and at 4 10 strides $\left(\lambda_{\mathrm{L}}\right)^{1,2}$

Orbital stability was estimated using maximum Floquet multipliers, ${ }^{1,7,14,27}$ which provide an indication of the rate of growth of small perturbations from one cycle to the next. For these calculations, the trajectories were first time normalized so that each stride contained exactly 101 samples, representing 101 Poincaré sections. Then, the Floquet multiplier for a chosen Poincaré section was calculated using the formula:

$$
\left[S_{k+1}-S^{*}\right]=J\left(S^{*}\right)\left[S_{k}-S^{*}\right]
$$

where $S_{k}$ is the value of the trajectory crossing at the Poincaré section during stride $k, S^{*}$ is the mean of all $S_{k}$, and $J$ is the Jacobian mapping in first order the deviation from $S^{*}$ of this trajectory crossing to its deviation from $S^{*}$ at its next crossing (i.e. its next cycle $S_{k+1}$ ). The maximum eigenvalue of $J$ represents the maximal Floquet multiplier. For further analysis, the largest Floquet multiplier across all 101 Poincaré sections, i.e., across all different phases in the stride cycle was used (MaxFm), ${ }^{7}$ as this represents the most unstable point in the stride cycle.

All calculations were performed using custom-made Matlab programs (The MathWorks, Inc., Natick, MA).

The effects of walking speed on the estimates of the stability measures from the two different measurement systems were tested by performing a repeated measures ANOVA. Since we assume that both measurement systems should yield similar results, significant effects of measurement system would indicate that absolute values obtained from different measurement systems (within a subject) cannot be compared, while a significant interaction would indicate that quantitative effects of manipulations (within a subject) cannot be compared between measurement systems. Of course, even if the latter is the case, qualitative effects of walking speed may still be similar across measurement systems. Thus, to further assess how well the estimates from both systems corresponded, we calculated the correlation $(R)$ between the outcomes of both systems. A high correlation would indicate that similar (qualitative) effects of experimental manipulations/subject group will be found, although quantitatively these effects may differ. This may be a problem when comparing data from different studies using different methodologies, but would still allow for comparison of experimental conditions/subject groups using the same methodology.

In case a low correlation $(R<0.8)$ between the two measurement systems was found for a given dependent measure, a post-hoc analysis was performed, in which 
the global Optotrak data were rotated to the local cluster marker coordinate system, and the measure was calculated from this data. To this end, the orientation matrix of the cluster marker was calculated in the global coordinate system for each time frame. Subsequently, to obtain the local angular velocity and linear acceleration vectors, the global angular velocity and linear acceleration vectors were pre-multiplied by the inverse of this orientation matrix. Comparing the measure as calculated from the rotated data to the measure as calculated from the original Optotrak data allowed us to separate the effect of instrumentation and the choice of reference frame.

\section{RESULTS}

We found a main effect of measurement system for $\lambda_{\mathrm{L}}$ only $(p=0.001)$, which was relatively small (see Fig. 3). More importantly, there were no significant speed $\times$ measurement system interactions for any of the measures, indicating that the effects of speed were not quantitatively different between measurement systems. Significant main effects of walking speed on $\lambda_{\mathrm{s}}$ and $\lambda_{\mathrm{L}}$ were present $\left(p<0.001\right.$ for both): $\lambda_{\mathrm{s}}$ decreased and $\lambda_{\mathrm{L}}$ increased with increasing walking speed. The main effect of walking speed on MaxFm was not significant ( $p=0.31$, see Fig. 3), although visual inspection of the data suggested that with increasing walking speed MaxFm first decreased slightly and then increased somewhat.

For $\lambda_{\mathrm{s}}$, the correlation coefficient between the two measurement systems was 0.87 , while for $\lambda_{\mathrm{L}}$ it was 0.98. For MaxFm, the correlation was the lowest, 0.66 (see Fig. 4). For MaxFm, when we compared the measure as calculated from "normal" and "rotated" Optotrak data, we found a much higher correlation, 0.97 .

\section{DISCUSSION}

Taken together, the results demonstrate that the investigated stability measures, especially $\lambda_{\mathrm{s}}$ and $\lambda_{\mathrm{L}}$,
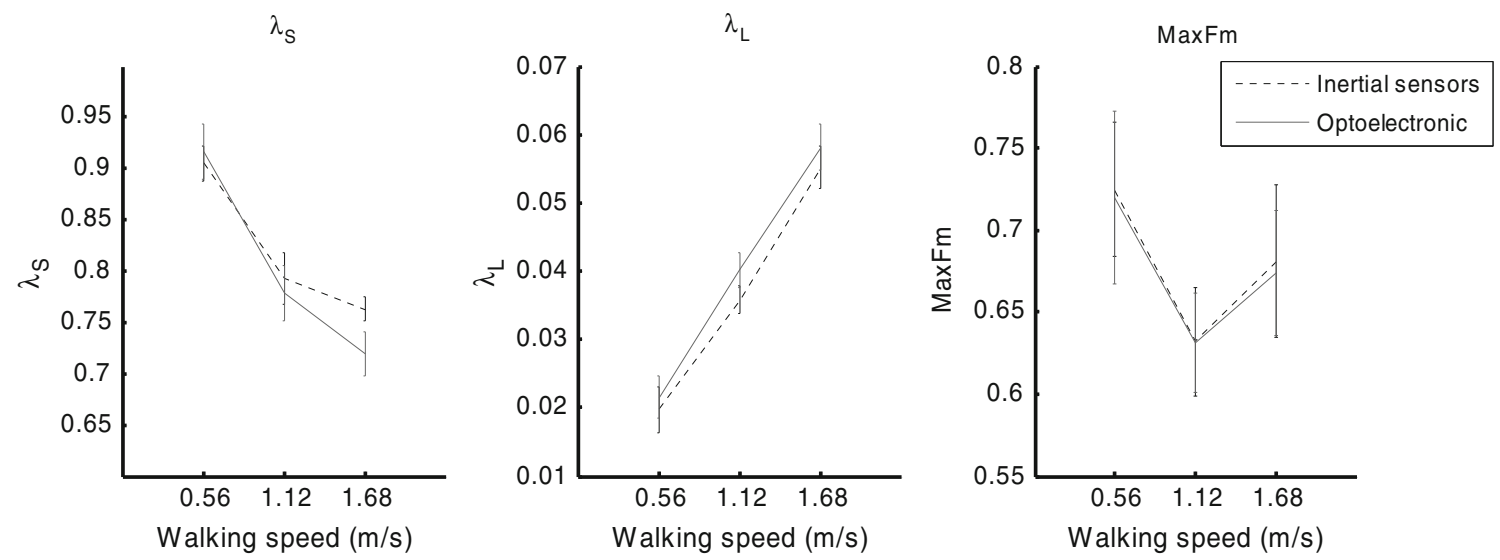

FIGURE 3. Effects of walking speed. Effects of walking speed on $\lambda_{\mathbf{S}}, \lambda_{\mathrm{L}}$ and MaxFm. Error bars represent standard errors.
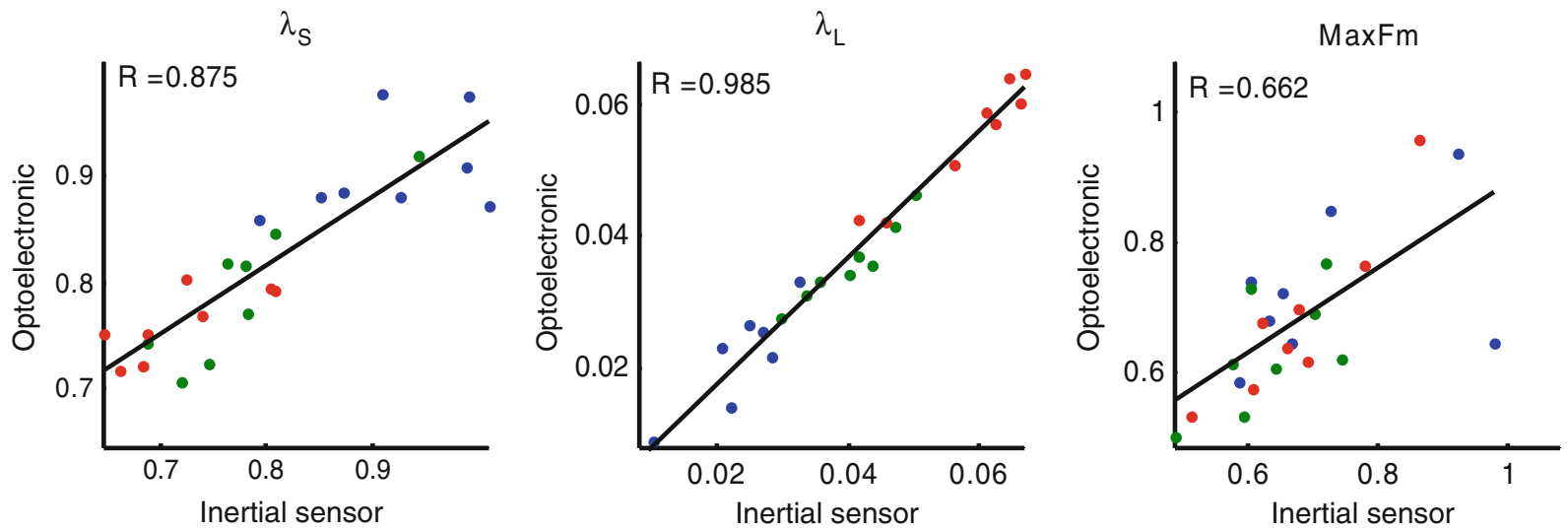

FIGURE 4. Correlation between measurement systems. The correlation between the two different measurement systems for $\lambda_{\mathbf{s}}$, $\lambda_{\mathrm{L}}$, and MaxFm. Blue $=0.56 \mathrm{~m} / \mathrm{s}$, green $=1.12 \mathrm{~m} / \mathrm{s}$, and $\mathrm{red}=1.68 \mathrm{~m} / \mathrm{s}$. 
may well be estimated using non-aligned inertial sensors. In comparing the two measurement systems, we found similar effects of walking speed on $\lambda_{\mathrm{S}}, \lambda_{\mathrm{L}}$, and MaxFm, indicating that the two measurement methods lead to comparable results and thus may be used interchangeably. It should be noted that these effects of walking speed, in particular those with regard to $\lambda_{\mathrm{s}}$, are in disagreement with some published findings, ${ }^{9,10}$ but consistent with more recent reports. ${ }^{2}$ In all likelihood, this discrepancy/consistency stem from the fact that we used an equal number of strides for all walking speeds, rather than an equal amount of time, as was done in some studies. ${ }^{1}$ Although the present study is not the first to report that $\lambda_{\mathrm{s}}$ and $\lambda_{\mathrm{L}}$ show opposite effects of walking speed, ${ }^{1,2,11}$ this finding is not yet well understood. It has been suggested that these measures may reflect different properties of gait stability. ${ }^{28}$ Still, how this may contribute to our understanding of speed on these measures remains to be investigated.

We found high correlations between the two systems for $\lambda_{\mathrm{s}}$ and $\lambda_{\mathrm{L}}$, but a lower correlation for MaxFm. The low variance of MaxFm with walking speed, as manifested by non-significant speed effects on this parameter, may be a reason for the lower correlation between the two measurement systems for MaxFm (i.e., if there would be no variance because of walking speed, there would be proportionally more error variance and the correlation would be lower, even if the measurement systems would not perform all that poorly in reality). Also the fact that the Poincare sections were not sampled at exactly the same time may have played a role, as this may affect the positions of the trajectories in the Poincaré section, especially when those trajectories are sharply curved. These kind of differences should become smaller with increasing sample frequency, or when analyzing the mean of all maximum Floquet multipliers within the stride cycle. However, in an extensive post-hoc analysis of the correlation of the mean of all maximum Floquet multipliers of all Poincaré sections within a stridecycle, we found no higher correlation between the two systems $(R=0.67)$, which speaks against this effect. All in all, the low correlation between the measurement systems that was found for MaxFm raises the question whether the chosen state space descriptions were sufficient to calculate this measure in the first place. However, the post-hoc analysis in which the rotated Optotrak were compared with the normal Optotrak data suggested that the differences between measurement systems (i.e., measurement noise) may explain most of the lack of correlation. Of course, this noise would be present for all measures, which suggests that MaxFm is more sensitive to measurement noise than $\lambda_{\mathrm{s}}$ and $\lambda_{\mathrm{L}}$, but may be calculated from data in different reference frames.
This potential sensitivity of MaxFm to measurement noise may be caused by the way it is calculated. To start with, $S^{*}$ is likely to be little affected by (Gaussian) noise, as it represents an average of all trajectory crossings. However, $J$ is calculated using a least squares solution to Eq. (2), and it may well be that noise has large effects on this solution, especially if the minimum of the solution is shallow. An in-depth analysis of these effects is outside the scope of the current paper, but should be addressed in future studies. Note that both $\lambda_{\mathrm{s}}$ and $\lambda_{\mathrm{L}}$ are based on averaging, which renders noise less of an issue, as was also shown by Rosenstein et al. ${ }^{25}$

The fact that $\lambda_{\mathrm{L}}$ showed the highest correlation between measurement systems may be caused by the fact that the rate of divergence after 1 stride will also be largely dependent upon the structure of stride time variability, ${ }^{2,16}$ which will be the same for both measurement systems.

The fact that inertial sensors may be used as a viable and valid alternative for optoelectronic measurement systems constitutes a considerable advantage in studies on gait stability. Inertial sensor systems are easier to use and cheaper than optoelectronic measurement systems and may thus be readily applied. This will allow for large-scale studies in which gait stability, perceived stability, and occurrence of falling are measured and correlated. These studies may then help to further establish the relationship between measures of dynamic gait stability and more real-life notions of stability. Once this relationship has been established, a window of opportunity is opened for clinical application of these measures in terms of, e.g., diagnostics, identification of fall prone subjects, and evaluation of treatment programs. Still, the usability of portable sensors remains to be demonstrated in more realistic conditions, such as overground walking, which differs in stability from treadmill walking. ${ }^{5}$

\section{ACKNOWLEDGMENTS}

This work was funded in part by a grant from Biomet Nederland. We are grateful to Joost Haeck for his assistance in collecting the data.

\section{OPEN ACCESS}

This article is distributed under the terms of the Creative Commons Attribution Noncommercial License which permits any noncommercial use, distribution, and reproduction in any medium, provided the original author(s) and source are credited. 


\section{REFERENCES}

${ }^{1}$ Bruijn, S. M., J. H. van Dieen, O. G. Meijer, and P. J. Beek. Statistical precision and sensitivity of measures of dynamic gait stability. J. Neurosci. Methods 178(2):327333, 2009.

${ }^{2}$ Bruijn, S. M., J. H. van Dieën, O. G. Meijer, and P. J. Beek. Is slow walking more stable? J. Biomech. 42(10): 1506-1512, 2009.

${ }^{3}$ de Vries, W. H., H. E. Veeger, C. T. Baten, and F. C. van der Helm. Magnetic distortion in motion labs, implications for validating inertial magnetic sensors. Gait Posture 29(4):535-541, 2009.

${ }^{4}$ Dingwell, J. B., and J. P. Cusumano. Nonlinear time series analysis of normal and pathological human walking. Chaos 10(4):848-863, 2000.

${ }^{5}$ Dingwell, J. B., J. P. Cusumano, P. R. Cavanagh, and D. Sternad. Local dynamic stability versus kinematic variability of continuous overground and treadmill walking. J. Biomech. Eng. 123(1):27-32, 2001.

${ }^{6}$ Dingwell, J. B., J. P. Cusumano, D. Sternad, and P. R. Cavanagh. Slower speeds in patients with diabetic neuropathy lead to improved local dynamic stability of continuous overground walking. J. Biomech. 33(10):1269-1277, 2000.

${ }^{7}$ Dingwell, J. B., and H. G. Kang. Differences between local and orbital dynamic stability during human walking. J. Biomech. Eng. 129(4):586-593, 2007.

${ }^{8}$ Dingwell, J. B., H. G. Kang, and L. C. Marin. The effects of sensory loss and walking speed on the orbital dynamic stability of human walking. J. Biomech. 40(8):1723-1730, 2007.

${ }^{9}$ Dingwell, J. B., and L. C. Marin. Kinematic variability and local dynamic stability of upper body motions when walking at different speeds. J. Biomech. 39(3):444-452, 2006.

${ }^{10}$ England, S. A., and K. P. Granata. The influence of gait speed on local dynamic stability of walking. Gait Posture 25(2):172-178, 2007.

${ }^{11}$ Fallah Yakhdani, H. R., H. Abbasi Bafghi, O. G. Meijer, S. M. Bruijn, N. van den Dikkenburg, A. B. Stibbe, B. J. van Royen, and J. H. van Dieen. Stability and variability of gait in knee osteoarthritis before and after replacement surgery. Clin. Biomech., 2010, epub.

${ }^{12}$ Gates, D. H., and J. B. Dingwell. Comparison of different state space definitions for local dynamic stability analyses. J. Biomech. 42(9):1345-1349, 2009.

${ }^{13}$ Granata, K. P., and T. E. Lockhart. Dynamic stability differences in fall-prone and healthy adults. J. Electromyogr. Kinesiol. 18(2):172-178, 2008.

${ }^{14}$ Hurmuzlu, Y., and C. Basdogan. On the measurement of dynamic stability of human locomotion. J. Biomech. Eng. 116(1):30-36, 1994.

${ }^{15}$ Hurmuzlu, Y., C. Basdogan, and D. Stoianovici. Kinematics and dynamic stability of the locomotion of post-polio patients. J. Biomech. Eng. 118(3):405-411, 1996.
${ }^{16}$ Jordan, K., J. H. Challis, J. P. Cusumano, and K. M. Newell. Stability and the time-dependent structure of gait variability in walking and running. Hum. Mov. Sci. 28(1): 113-128, 2009.

${ }^{17}$ Kang, H. G., and J. B. Dingwell. A direct comparison of local dynamic stability during unperturbed standing and walking. Exp. Brain Res. 172(1):35-48, 2006.

${ }^{18}$ Kang, H. G., and J. B. Dingwell. Intra-session reliability of local dynamic stability of walking. Gait Posture 24(3):386390, 2006

${ }^{19}$ Kang, H. G., and J. B. Dingwell. Effects of walking speed, strength and range of motion on gait stability in healthy older adults. J. Biomech. 41(14):2899-2905, 2008.

${ }^{20}$ Kang, H. G., and J. B. Dingwell. Dynamic stability of superior vs. inferior segments during walking in young and older adults. Gait Posture 30(2):260-263, 2009.

${ }^{21}$ Kantz, H., and T. Schreiber. Nonlinear Time Series Analysis. Cambridge Nonlinear Science Series 7, Cambridge, New York: Cambridge University Press, xvi, 304 p., 1997.

${ }^{22}$ Manor, B., and L. Li. Characteristics of functional gait among people with and without peripheral neuropathy. Gait Posture 30(2):253-256, 2009.

${ }^{23}$ Mees, A. I., and K. Judd. Dangers of geometric filtering. Physica D 68(3-4):427-436, 1993.

${ }^{24}$ Roetenberg, D., H. J. Luinge, C. T. M. Baten, and P. H. Veltink. Compensation of magnetic disturbances improves inertial and magnetic sensing of human body segment orientation. IEEE Trans. Neural Syst. Rehab. Eng. 13(3): 395-405, 2005.

${ }^{25}$ Rosenstein, M. T., J. J. Collins, and C. J. Deluca. A practical method for calculating largest Lyapunov exponents from small data sets. Physica D 65(1-2):117-134, 1993.

${ }^{26}$ Rubenstein, L. Z. Falls in older people: epidemiology, risk factors and strategies for prevention. Age Ageing 35(Suppl 2):ii37-ii41, 2006.

${ }^{27}$ Schablowski, A., and H. J. Gerner. Comparison of two measures of dynamic stability during treadmill walking. Fast Motions in Biomechanics and Robotics: Optimization and Feedback Control, vol. 340, pp. 345-360, 2006.

${ }^{28} \mathrm{Su}$, J. L. S., and J. B. Dingwell. Dynamic stability of passive dynamic walking on an irregular surface. J. Biomech. Eng. 129(6):802-810, 2007.

${ }^{29}$ van Acht, V., E. Bongers, N. Lambert, and R. Verberne. Miniature wireless inertial sensor for measuring human motions. Proceedings of the 29th I.C. IEEE EMBC, Lyon, France, 2007.

${ }^{30}$ Yoshino, K., T. Motoshige, T. Araki, and K. Matsuoka. Effect of prolonged free-walking fatigue on gait and physiological rhythm. J. Biomech. 37(8):1271-1280, 2004.

${ }^{31}$ Zatsiorsky, V. M. Kinematics of Human Motion. Champaign, IL: Human Kinetics, 1998. 